\title{
Fatal, spontaneous, unknown aortal aneurysm dissection in a woman of 38 weeks' gestation
}

BY JASNA ČERKEZ HABEK, DUBRAVKO HABEK, ELIZABETA HORVATIĆ, SMILJKA BEHIN

\section{Abstract}

We describe a case of spontaneous dissection of an unknown ascending thoracic aortic aneurysm in a 35year-old woman in her $38^{\text {th }}$ week of pregnancy, resulting in an atypical clinical picture, emergency caesarean section, and lethal outcome in both the mother and child.

Key words: aortic dissection, pregnancy, caesarean section, perinatal death

\section{Introduction}

Acute aortic syndrome (AAS) is a possible non-obstetric cause of high maternal and fetal mortality. The most common feature of AAS is aortic dissection (AD), which occurs usually due to trauma, but may also be spontaneous due to pathomorphological lesions of the aortic wall, 
hypertension, collagenosis, Marfan syndrome, syphilis, etc. (1-4) The most common location of $\mathrm{AD}$ is the ascending aorta (Stanford type $\mathrm{A}$ ), in $90 \%$ of cases located within $10-15 \mathrm{~cm}$ of the aortic valve; dissections generally originate from intimal tears supported by hypertension or rupture of vasa vasorum. (4) Chest pain is the most frequent symptom of acute $\mathrm{AD}$, with abrupt onset of severe chest and/or back pain being the most typical feature. After acute aortic rupture, aortic regurgitation is the second most common cause of death in patients with AD. (5) Patients with acute severe aortic regurgitation commonly present with heart failure and cardiogenic shock. Surgery is the treatment of choice for acute type $\mathrm{A} \mathrm{AD}$; mortality is $50 \%$ within the first 48 hours if not operated. Despite improvements in surgical and anaesthetic techniques, perioperative mortality (25\%) and neurological complications (18\%) remain high. (6)

We describe a case of spontaneous $\mathrm{AD}$ type $\mathrm{A}$ in a pregnant woman in her $38^{\text {th }}$ week of pregnancy, who presented with an atypical clinical picture resulting in an emergency caesarean section, and a lethal outcome in both the mother and the child.

\section{Case presentation}

A 35-year-old woman, in her $38^{\text {th }}$ week of pregnancy, was admitted to the emergency room for mild chest pain, breathing difficulty, vomiting and diarrhoea lasting three days, with the onset of intensive chest pain radiating to the back one hour before admission. Otherwise, she had been suffering from epilepsy for two years and taking lamotrigine, $2 \times 100$ $\mathrm{mg}$. At the age of 27 she had undergone conization for severe cervical dysplasia (CIN III). At the age of 29, she had a caesarean section due to fetal hypoxia and delivered a live female, 2900/51, now a healthy child. In the meantime, she also had one spontaneous abortion. The course of her current pregnancy was normal, with the exception of gestational diabetes treated by diabetic diet, with normal glucose levels. Her family history revealed that her father had undergone cardiac valve surgery and died from sepsis postoperatively; there was no hypertensive disease or 
collagenosis in her family history. On admission to the emergency room, the patient was conscious, communicable, oriented, eupnoeic and afebrile. On examination, normal breath sounds, rhythmic heart action and clear heart sounds were recorded, no murmur; the abdomen was soft, non-tender, with audible peristalsis, no palpable visceromegaly or peritonism; blood pressure 120/70; pulse 68 ; respiration 18; oxygen saturation $\left(\mathrm{SpO}_{2}\right)$ 100; pain by visual analogue scale $5 / 10$; electrocardiogram (ECG): sinus rhythm, action 75/min. Laboratory findings were normal (haemogram, leukogram and biochemistry). Metoclopramide $20 \mathrm{mg}$ in infusion of $500 \mathrm{~mL}$ physiologic solution was prescribed for digestive discomforts and a working diagnosis of gastroenterocolitis was made. Thirty minutes following admission, the patient was seen by the consultant gynaecologist who described an adipose woman in her $38^{\text {th }}$ week of pregnancy with a normal ultrasound, anxious, with vomiting and mild epigastric pain, normal reactive 30minute cardiotocography (CTG) recording without contractions, blood pressure 110/70, pulse 75/min. Gynaecologic finding: shortened cervix, cervical canal patent one finger width to the membrane and fetal head. Immediately prior to the planned magnetic resonance imaging (MRI), one hour following admission, the patient complained of nausea, vomiting and difficulty breathing again. An abrupt tonic-clonic seizure, with loss of consciousness, occurred on trying to lift her to a sitting position and intravenous (i.v.) diazepam, $10 \mathrm{mg}$, with oxygen was prescribed for the clinical picture of grand mal epileptic attack, which was immediately followed by cardiorespiratory arrest, with unmeasurable blood pressure and pulse. Cardiorespiratory resuscitation with endotracheal intubation was initiated; CTG showed bradycardia, thus urgent caesarean section was performed to deliver a female neonate without vital signs; the neonate was unsuccessfully resuscitated and death was declared after 1-hour of intensive resuscitation. Intraoperatively, no other pathological findings or haemoperitoneum were found in the abdominal cavity. Cardiorespiratory recovery failed to occur during the procedure with continuous simultaneous resuscitation, and the patient was declared dead. Of the haemogram and coagulogram 
findings repeated during the procedure and resuscitation, only D-dimers were elevated (6689.68), and they were the first laboratory sign of possible AAS or pulmonary embolism.

The woman's and neonate's bodies were referred for autopsy under the diagnosis of refractory cardiorespiratory arrest and sudden death, along with possible obstetric embolism. Among other pathomorphological findings, the autopsy report pointed to the following: fresh horizontal suprapubic surgical incision, $17 \mathrm{~cm}$ long, with 7 visible sutures; external genitalia of normal appearance; both lungs of normal volume, heavy; on section, pleura moist, heavy and softened, at the cutting plane dark-red to violet in colour; smooth parenchyma, with some foamy reddish fluid and abundant blood drained upon pressure; $500 \mathrm{cc}$. of fluid and $400 \mathrm{cc}$. of coagulated blood found in the pericardium; the inner pericardium surface and outer wall of the heart smooth and shiny; the heart measuring 11x12x5 cm; right heart cavities slightly enlarged, ventricular thickness $5 \mathrm{~mm}$; foramen ovale closed; cardiac valves thin, transparent, of normal appearance; pulmonary artery of smooth intima, $65 \mathrm{~mm}$ in diameter; the myocardium light-brown, moist and flaccid; left heart cavities slightly enlarged, ventricular thickness $15 \mathrm{~mm}$; proximal aorta measuring $66 \mathrm{~mm}$, with smooth and grayish intima; intimal inverse $\mathrm{T}$ shaped rupture of the ascending segment of thoracic aorta found $12 \mathrm{~mm}$ above the aortic valve base posterolaterally on the left, horizontal length $3.8 \mathrm{~cm}$ and vertical length $1.4 \mathrm{~cm}$; locally, intramural haematoma, $3 \mathrm{~cm}$ wide and $7 \mathrm{~cm}$ long, extending to the aortic arch and to a minor part of the descending thoracic aorta; vertical rupture of the aortic adventitia, $0.4 \mathrm{~cm}$ in length, found above the mentioned intimal rupture; the rest of aorta free from lesions; coronary artery of medium width, patent, with smooth intima. Histology indicated a lesion between the external and medium third of media, associated with moderate atherosclerotic lesions (atherosclerosis gradus medii). Uterus altered by gravidity, vagina with

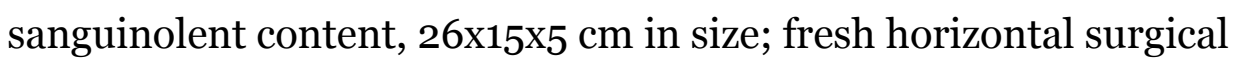
incision, $8 \mathrm{~cm}$ in length, with 10 visible sutures on the anterior wall of the lower aspect of the body of uterus; uterine tube and ovaries of normal morphology. 
The following was stated as the cause of death: ascending thoracic aortal aneurysm dissection with haematoma and haematopericardium. Autopsy of the neonate's body showed normal morphology with pronounced cyanosis and petechial haemorrhage on serous membranes, indicating peracute asphyxia.

\section{Discussion}

$\mathrm{AD}$ is very rare in pregnancy, with only a few cases reported in the literature. It is usually unrecognized and associated with high perinatal mortality, with $\mathrm{AD}$ demonstrated as the cause of sudden death only at autopsy. (7) Fatal cases of visceral aneurysm dissection, e.g., splenic artery dissection, (8) are even rarer. Nassiel et al. investigated the incidence of $\mathrm{AD}$ in Sweden during the 1999-2007 period and found it to be $14.5 / 1,000,000$ with maternal mortality of 4.4/1,000,000 pregnancies. During the study period, four women died and one woman survived, with one stillborn and four asphyxial newborns. (3)

The haemodynamic events in pregnancy, delivery and puerperium are known to increase the risk of aortic dissection, intracerebral haemorrhage and visceral, e.g., splenic artery dissection. (1,9) Marfan syndrome has been described as a possible cause of aortic dissection with a picture of abrupt cardiovascular collapse in pregnancy and potential fatal outcome. (10) Panczel et al. have described sudden death in a multipara due to spontaneous ascending AA dissection, with a positive family history of sudden deaths in pregnancy, suggesting the potential genetic basis of AA. (11) Townend et al. report on sudden death of a pregnant woman in her third trimester, referred to a regional cardiothoracic hospital for chest pain and detection of a thoracic aortic aneurysm, probably secondary to an injury. The patient died on the way to hospital, and autopsy revealed rupture of a saccular thoracic AA. The authors concluded that haemodynamic changes occurring in pregnancy may lead to rupture of a previously silent, traumatic AA. (2) There are also rare cases described in the literature of sudden death in the puerperium, without a known positive personal and/or family history of 
ascendant aorta, where pathomorphological causes were found in the aortic arch (acute dissection type B according to Stanford) or descending aorta, also detected on autopsy. $(12,13)$

In the case of pregestationally diagnosed AA, termination of pregnancy and delivery should always be performed in clinical hospitals with complete obstetric, neonatologic, cardiovascular and resuscitation logistics in order to reduce the very high risk of maternal mortality. Snir et al. have described two cases of $\mathrm{AD}$ in a pregnant woman and puerpera without Marfan syndrome, where they performed corrective vascular surgery and caesarean section that resulted in both maternal and neonatal survival. (14) Beirer et al. have recently reported favourable outcomes and survival in a 34-year-old mother and her newborn after urgent caesarean section and emergency replacement of the ascending aorta and aortic arch due to AA dissection extending $1 \mathrm{~cm}$ distally from the aortic valve over the full length up to the iliac arteries, involving the brachiocephalic and carotid arteries up to the level of the larynx. (4)

However, it appears that such very rare cases of undetected, asymptomatic AA with sudden death will continue to be anecdotal and tragic for medical staff and those young women's families. In our case, we describe the dissection of an unknown and asymptomatic aneurysm of the thoracic aorta, with haemopericardium and lethal outcome for the patient and her newborn. Cardiothoracic surgery for this acute event could not be performed for logistical reasons because the delivery occurred in a secondary obstetric institution in a general hospital.

\section{References}

1. Lapinsky SE, Kruczynski K, Slutsky AS. Critical Care in the Pregnant Patient. Am J Respir Crit Care Med 1995;152:427-55.

2. Townend JN, Davies MK, Jones EL. Fatal rupture of an unsuspected post-traumatic aneurysm of the thoracic aorta during pregnancy. $\mathrm{Br}$ Heart J 1991;66(3):248-9.

3. Nasiell J, Norman M, Lindqvist PG, Malmstedt J, Bottinga R, Blennow R. 
Aortic dissection in pregnancy: a life-threatening disease and a diagnosis of worth considering. Acta Obstet Gynecol Scand 2009;88(10):1167-70.

4. Beirer M, Banke IJ, Münzel D, Wendorff H, Khaladj N, Kirchhoff C, Huber-Wagner S, Biberthaler P. Emergency cesarean section due to acute aortic dissection type A (Debakey I) without Marfan syndrome: a case report and review of the literature. J Emerg Med 2014;46(1)13-7.

5. Erbel R, Oelert H, MeyerJ, Puth M, Mohr-Katoly S, Hausmann D, et al. Effect of medical and surgical therapy on aortic dissection evaluated by transesophageal echocardiography. Implications for prognosis and therapy. The European Cooperative Study Group on Echocardiography. Circulation 1993;87:1604-15.

6. Gilon D, Mehta RH, Oh JK, Januzzi JL Jr., Bossone E, Cooper JV, et al. Characteristics and in-hospital outcomes of patients with cardiac tamponade complicating typeAacute aortic dissection. Am J Cardiol 2009;103:1029-31.

7. Monrozies M, Bouissou H, Grynfogel M. Fatal rupture of an abdominal aortic aneurysm during pregnancy. Bull Fed Soc Gynecol Obstet Lang Fr 1962;14:791-5.

8. Hunsaker DM, Turner S, Hunsaker JC 3rd. Sudden and unexpected death resulting from splenic artery aneurysm rupture: two case reports of pregnancy-related fatal rupture of splenic artery aneurysm. Am J Forensic Med Pathol 2002;23(4):338-41.

9. Barrett JM, Van Hooydonk JE, Boehm FH. Pregnancy-related rupture of arterial aneurysms. Obstet Gynecol Surv 1982;37:557-66.

10. Sutinen S, Piiroinen O. Marfan syndrome, pregnancy, and fatal dissection of aorta. Acta Obstet Gynecol Scand 1971;50(3):295300.

11. Pánczél Z, Sára L, Tóth P, Hubay M, Keller E, Langmár Z, Pajor A. Spontaneous aortic rupture during pregnancy. Orv Hetil 2011;152(23):929-33.

12. Ichigo S, Sugiyama M, Murase T, Ikeda T, Imai A.An autopsy case of acute aortic dissection during postpartum period. Clin Exp Obstet Gynecol 2013;40(3):435-6.

13. Monteiro FN, Bhagavath P, Rao L, Pai ND, Kanchan T, Menezes RG, et al. Descending thoracic aortic aneurysm rupture during postpartum 
period. J Forensic Sci 2011;56(4):1054-7.

14. Snir E, Levinsky L, Salomon J, Findler M, Levy MJ, Vidne BA.

Dissecting aortic aneurysm in pregnant women without Marfan disease.

Surg Gynecol Obstet 1988;167(6):463-5.

Jasna Čerkez Habek

University Department of Internal Diseases,Cardiovascular Unit, University Hospital „Sveti Duh“, Croatian Catholic University Zagreb, Croatia

Dubravko Habek

University Department of Obstetrics and Gynaecology, University Hospital „Sveti Duh“,

Croatian Catholic University Zagreb, Croatia

Elizabeta Horvatić

Department of Pathology, General Hospital Koprivnica, Croatia

Smiljka Behin

Gynaecology and Obstetrics, General Hospital Koprivnica, Croatia

Correspodence address:

Jasna Čerkez Habek

Department of Internal Diseases,Cardiovascular Unit

University Hospital „Sveti Duh“, Zagreb

Sveti Duh 64, 10000 Zagreb, Croatia

E-mail: jasna.habek@gmail.com

Article printed from Signa Vitae: http://www.signavitae.com

URL to article: http://www.signavitae.com/2016/o5/fatalspontaneous-unknown-aortal-aneurysm-dissection-in-awoman-of-38-weeks-gestation/

Copyright (C) 2015 Signa Vitae. All rights reserved. 